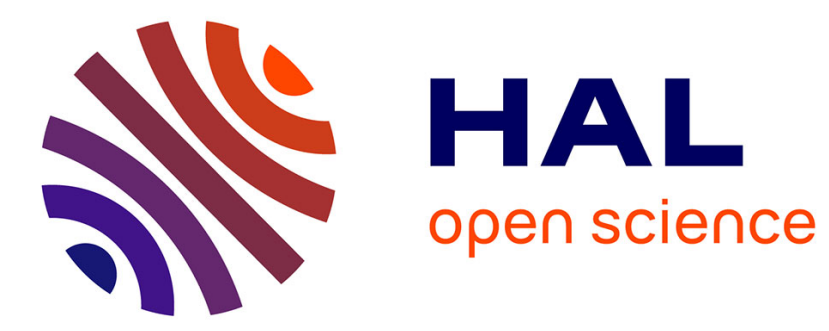

\title{
Joseph Wresinski : pionnier de l'empowerment radical made in France
}

\author{
Alex Roy
}

\section{To cite this version:}

Alex Roy. Joseph Wresinski: pionnier de l'empowerment radical made in France. Mouvements: des idées et des luttes, 2016, 1 (85), pp.87-94. 10.3917/mouv.085.0087 . hal-02329015

\section{HAL Id: hal-02329015 \\ https://hal.science/hal-02329015}

Submitted on 23 Oct 2019

HAL is a multi-disciplinary open access archive for the deposit and dissemination of scientific research documents, whether they are published or not. The documents may come from teaching and research institutions in France or abroad, or from public or private research centers.
L'archive ouverte pluridisciplinaire HAL, est destinée au dépôt et à la diffusion de documents scientifiques de niveau recherche, publiés ou non, émanant des établissements d'enseignement et de recherche français ou étrangers, des laboratoires publics ou privés. 


\section{Joseph Wresinski :}

\section{Pionnier de l'empowerment radical made in France}

\section{Alex Roy, EVS-RIVES, Université de Lyon, CNRS UMR 5600}

Version auteur de l'article suivant : ROY A., 2016, « Joseph Wresinski : pionnier de l'empowerment radical made in France », Mouvements, vol. 1, n85, p. 87-94.

L'empowerment radical peut être défini comme une stratégie d'émancipation collective et politique. Cette émancipation se caractérise par un processus collectif de prise de conscience du monde environnant et de la place que l'on y occupe ainsi que par le développement d'une volonté d'agir pour transformer ce monde à son échelle. "Dans cette optique, les enjeux de l'empowerment sont la reconnaissance, l'autodétermination, la redistribution des ressources et les droits politiques ${ }^{1} \gg$. Dans ce modèle qui se décline en différents modes d'action, Saul Alinsky et Paulo Freire constituent deux figures incontournables. Ces deux contemporains sont internationalement reconnus. Nombre d'initiatives citoyennes s'inspirent de la pédagogie freirienne ou des principes d'action alinskiens. À peu près à la même époque, Joseph Wresinski est à l'origine d'un mouvement qui développe une stratégie d'émancipation qui leur est proche. Il s'agit du mouvement international de lutte contre la pauvreté ATD Quart Monde, créé en France à la fin des années 1950.

Contrairement à Freire et Alinsky, le mouvement de Wresinski ne constitue pas une référence ${ }^{2}$ : très peu d'organisations s'en revendiquent et les chercheurs ne s'y attardent que rarement. Cette association est souvent perçue comme un mouvement consensuel sans véritable portée subversive ${ }^{3}$. À partir d'une enquête de terrain sur le groupe local d'ATD Quart Monde Rhône-Alpes, d'entretiens réalisés auprès de responsables nationaux et d'un travail bibliographique sur le Père Joseph Wresinski, cet article montre pour autant que ce mouvement, même s'il n'en revendique pas le label, peut être envisagé comme un pionnier de l'empowerment radical français. Le projet de société d'ATD Quart Monde défend une révolution culturelle à partir des plus démunis ainsi qu'un renouvellement démocratique visant la justice sociale. Nous verrons également qu'ATD Quart Monde conserve une radicalité politique tout en valorisant les notions de coopération et d'alliance.

1 M.-H. Bacqué et C. Biewener, L’empowerment, une pratique émancipatrice, La Découverte, Paris, 2013, p.15.

2 Le modèle ATD Quart Monde commence aujourd'hui à devenir une référence à l'étranger, comme le suggère le mouvement québécois Paroles d'excluEs qui s'en inspire directement.

3 C'est par exemple la vision de F. Viguier, «Lutter pour les droits des exclus », dans B. Frère, M. Jacquemain (dirs.), Résister au quotidien?, Les presses de Science Po, Paris, 2013, p. 87-110. 


\section{La révolution culturelle à partir des plus pauvres}

Le projet de société défendu par Joseph Wresinski s'inscrit dans le courant de l'éducation populaire. L'action culturelle constitue ainsi un pivot dans la lutte contre l'exclusion proposée par ATD Quart Monde $^{4}$, une philosophie congruente avec la vision d'un Paulo Freire qui propose une révolution culturelle pour sortir les «opprimés» de la «culture du silence» régnant, à son époque, dans la dictature brésilienne ${ }^{5}$. En effet, Joseph Wresinski prône une révolution culturelle à partir de la revendication identitaire de ce qu'il appelle le «peuple du quart monde » c'est-à-dire le milieu de la grande pauvreté. Par une action fondée sur la connaissance, il s'agit de développer une conscience de classe dans le but de créer des « militants Quart Monde ${ }^{6} »$ réflexifs et critiques qui peuvent trouver une place dans la société tout en souhaitant la transformer. Pour Wresinski, cette révolution culturelle a pour but de mettre en lumière l'existence et de dénoncer la persistance de la misère extrême dans nos sociétés démocratiques occidentales.

Cependant, Wresinski se démarque de Freire au sens où son projet se construit sur la notion d'alliance avec des personnes «intégrées dans la société » et n'entre pas dans un antagonisme entre les différentes catégories sociales qui la composent. Pourtant, pour Freire, même si la neutralité est impossible, les « opprimés » comme les « oppresseurs » ont besoin d'être libérés. Joseph Wresinski va donc jusqu'au bout du raisonnement freirien en proposant une émancipation collective entre des personnes issues de différentes classes sociales. Pour cela, ATD Quart Monde propose d'aller «à la rencontre » des « exclus » en développant une relation de confiance sur la durée, car ils n'ont pas forcément conscience de la nécessité de s'émanciper. Les « inclus », quant à eux, doivent venir d'eux même dans une démarche volontaire d'émancipation.

Cette stratégie d'alliance est souvent à l'origine de l'image consensuelle dont subit le mouvement. Nous verrons que celle-ci n'empêche pas le mouvement d'être subversif, mais nous pouvons déjà noter que l'objectif de cette alliance est la lutte contre l'extrême pauvreté. Ainsi, Joseph Wresinski donne bien la priorité aux plus faibles et n'hésite pas à dénoncer la responsabilité des classes favorisées dans l'exclusion des plus pauvres. Le prêtre se retrouve alors sur ce point avec Paulo Freire $^{7}$. En effet, les pensées de Freire et de Wresinski s'appuient toutes les deux sur un sentiment d'indignation face à la pauvreté. Cependant, si Paulo Freire est un néomarxiste qui s'inscrit dans la lignée de la théologie de la libération, Joseph Wresinski, quant à lui, critique le marxisme. Selon lui, cette idéologie condamne les plus pauvres au silence en niant leur capacité à s'organiser.

\footnotetext{
${ }^{4}$ B. Lahire, L'invention de l'« illettrisme », La Découverte, Paris, 1999.

${ }^{5}$ P. Freire, Pédagogie des opprimés, La Découverte, Paris, 2001.

${ }^{6}$ Terme utilisé par le mouvement pour désigner les militants issus du milieu de la grande pauvreté.

${ }^{7}$ O. Naouar le décrit comme un défenseur de la cause des pauvres dans Paulo Freire, L'Harmattan, Paris, 2014.
} 
Le sentiment d'indignation de Wresinski prend ses racines dans son expérience personnelle de la misère durant l'enfance. Ensuite, il participe au mouvement des prêtres ouvriers dans lequel il se sent mis de côté à cause de son obsession du « sous-prolétariat». Il développe alors la volonté de défendre les personnes vivant dans la misère, un «peuple» qui semble délaissé de tous. En 1957, le Père Wresinski est envoyé dans le camp de Noisy-le-Grand. Face à la misère qu'il trouve dans ce ghetto, il décide de créer son propre mouvement pour défendre une transformation de la société dans son ensemble à partir des plus faibles : "Contre ce savoir qui donne d'une main la justice, pour la reprendre de l'autre, contre ce savoir introduit «par le haut» si l'on peut dire, il n'existe qu'un seul remède : le savoir introduit par le plus bas, le savoir montant dans la société comme un ballon d'oxygène, le savoir d'émulation, celui des chances égales ${ }^{8}$.»

Pour mettre en pratique cette conception, ATD Quart Monde crée, dans les années 1970, un dispositif participatif citoyen appelé 1' " université populaire ». Ce dispositif peut être défini comme une école de la citoyenneté fondée sur un processus proche de la «conscientisation » freirienne. Premièrement, des animateurs organisent des groupes de réflexion dans une optique de renforcement collectif des participants du «quart monde ». Deuxièmement, ces groupes se réunissent en plénière pour présenter leurs réflexions à des invités. Sur le long terme, l'objectif est de développer la volonté de transformer le monde. Puis, dans les années 2000, ATD Quart Monde crée les co-formations par le croisement des savoirs et des pratiques, un espace commun de réflexions et de propositions entre des personnes issues de la grande pauvreté et des professionnels (travailleurs sociaux, magistrats, personnels de la santé, chercheurs, politiques, etc.). Les co-formations sont alors conçues comme un prolongement de l'université populaire puisqu'elles donnent la possibilité aux «militants Quart Monde»d'agir concrètement pour transformer le monde à leur échelle : «Les universités populaires ça m’a permis de devenir la militante que je suis aujourd'hui, mais avec cette coformation, j'ai vraiment eu l'impression d'être utile en faisant bouger la vision du juge qui était en face de moi ${ }^{9}$. N Notons néanmoins que ces deux dispositifs sont centrés sur le développement des personnes et que leurs conséquences politiques restent limitées. Il serait alors intéressant d'analyser de quelle manière les réflexions provenant des universités populaires et des coformations - ce «savoir par le plus bas » - peuvent avoir des répercussions sur les campagnes politiques du mouvement.

Conformément à l'empowerment radical, Joseph Wresinski promeut ainsi une révolution culturelle à partir des plus pauvres. Il se différencie sur ce point de Saul Alinsky dont le projet n'a pas de dimension culturelle et qui cherche à organiser la société civile dans son ensemble sans donner la priorité aux plus pauvres. En effet, pour organiser les couches populaires, Saul Alinsky cherche l'efficacité. Selon Bruno Tardieu, ancien délégué national d'ATD Quart Monde, les «sous-

\footnotetext{
${ }^{8}$ J. Wresinski, Refuser la misère, Cerf, Paris, 2007, p.106.

${ }^{9}$ Militante quart monde, entretien, 23 octobre 2015.
} 
prolétaires " se caractérisant par une faible capacité à se mettre en mouvement d'eux-mêmes, la méthodologie d'Alinsky tend à les laisser de côté au profit des plus forts des quartiers populaires ${ }^{10}$. De plus, Alinsky voit même dans la classe moyenne une catégorie sociale cruciale pour gagner des victoires : «Le pouvoir et le peuple se trouvent dans la majorité dominante qu'est la classe moyenne ${ }^{11}$. » Il propose alors de concentrer les efforts d'organisation vers cette majorité en s'appuyant sur ses intérêts et ses problèmes. Si Joseph Wresinski voit également dans la classe moyenne des alliés essentiels, il entend, pour sa part, les réunir autour des problèmes des « sous-prolétaires ». Cependant, Joseph Wresinski partage avec Saul Alinsky une posture pragmatique radicale pour défendre une révolution démocratique.

\section{La révolution démocratique par le pragmatisme radical}

Comme Saul Alinsky, Joseph Wresinski défend un idéal démocratique visant la justice sociale. Dans la perspective de tendre vers cette utopie, ces deux contemporains prônent une révolution démocratique en redonnant du pouvoir politique à ceux qui en ont le moins. Pour ce faire, Alinsky entend sortir les citoyens ordinaires de leur apathie politique en développant leur leadership et leur rôle de contre-pouvoir. Joseph Wresinski et son mouvement s'efforcent, quant à eux, de développer la citoyenneté des « exclus » afin de leur donner une place sur la scène politique.

Contrairement à l'image consensuelle souvent attribuée à ATD Quart Monde, Joseph Wresinski est animé par une posture critique et radicale. S'il est vrai que le mouvement ne s'attaque pas de manière frontale au système capitaliste, il dénonce néanmoins les formes de domination qui lui sont liées en prônant une transformation radicale de la société vers un idéal sans exclusion. À l'instar de Saul Alinsky, Joseph Wresinski s'oppose ainsi vigoureusement à la logique de l'assistanat dominante. Selon lui, la société propose aux pauvres « essentiellement des relations d'aide individuelle sans autre contrepartie que d'en faire l'usage qu'elle impose ; elle leur crée une situation de dépendance personnelle sans retour. Refoulés dans la marge des inutiles, sans droits ni devoirs collectifs, ils sont privés des moyens de communication indispensables à l'acquisition d'une utilité quelconque ${ }^{12} »$. Par ailleurs, dans les années 1970, Joseph Wresinski adopte une vision innovante de la misère en tant que violation totale des droits de l'homme. Selon lui, l'analyse des conditions de vie des «sousprolétaires » permet de repérer et de comprendre les obstacles à chacun des droits et donc de faire avancer la lutte pour les droits de l'homme dans son ensemble. Le projet politique et pédagogique de Joseph Wresinski est alors de défendre les droits des plus démunis en leur donnant les moyens de les

\footnotetext{
${ }^{10}$ B. Tardieu, entretien, 20 avril 2014.

${ }^{11}$ S.D. Alinsky, Etre radical, Aden, Bruxelles, 2011, p.246.

12 J. Wresinski, op. cit., 2007, p. 32.
} 
faire valoir. Contrairement à l'approche de l'advocacy du courant de la défense des droits civiques aux États-Unis, ATD Quart Monde n'entend pas parler au nom des personnes vivant dans la misère, mais veut les accompagner pour leur faire «monter les marches de l'Élysée, du Vatican, de l'ONU ${ }^{13} »$. Dans la continuité de la pensée de Wresinski, le projet de société actuel d'ATD Quart Monde se résume dans leur théorie du «croisement des savoirs », une démarche de recherche-action qui vise à valoriser le savoir d'usage des personnes vivant dans la grande pauvreté par la confrontation avec les savoirs professionnels et universitaires, en affirmant que la société ne peut se construire démocratiquement sans cette expérience ${ }^{14}$.

Pour mettre en œuvre leur révolution démocratique, Joseph Wresinski et Saul Alinsky se retrouvent également autour de la posture pragmatique radicale. Hélène Balazard résume le pragmatisme radical alinskien comme une posture qui ne se focalise pas sur l'idéologie, mais sur la pratique dans une réflexivité permanente afin de lier la fin et les moyens ${ }^{15}$. Pour Joseph Wresinski, l'outil principal dans la lutte contre la pauvreté est la connaissance, à la fois par son caractère émancipateur pour les pauvres et par sa capacité à informer et à guider la société dans son ensemble. Dans cette optique, Joseph Wresinski rejette les dogmes idéologiques et adopte une posture réflexive. En effet, les membres de son mouvement peuvent être conçus comme des militants-anthropologues de la grande pauvreté qui programment leur action à partir d'une capitalisation de l'expérience vécue des personnes du «Quart Monde ». Joseph Wresinski avait le souci de «fonder et développer cette "science de la pauvreté" que nous [ATD Quart Monde] estimons une des armes principales du combat ${ }^{16} »$. ATD Quart Monde souhaite ainsi devenir un acteur du monde de la recherche pour pouvoir influencer les questions qu'il se pose. La recherche est également conçue comme un outil dans les campagnes politiques ou encore, dans une optique praxéologique, pour évaluer l'action du mouvement. Pour cela, le mouvement se dote d'outils spécifiques comme un institut de recherche et une maison d'édition. L'un des objectifs de l'institut de recherche est par exemple d'étudier la pensée de Wresinski pour l'actualiser avec les évolutions sociétales ${ }^{17}$.

En se démarquant des pratiques de lutte contre la pauvreté dominantes dans les années 1950, Joseph Wresinski est ainsi à l'origine d'un mouvement pionnier en France. La grande originalité de Joseph Wresinski, par rapport à ses deux contemporains que sont Paulo Freire et Saul Alinsky, est de proposer un projet qui concerne l'ensemble de la société à partir d'un rapprochement entre ceux qui n'ont pas de pouvoir d'un côté et ce qu'Alinsky nomme l'establishment de l'autre. Pour autant, le mode d'action d'ATD Quart Monde ne renonce pas au conflit et à l'idée de contre-pouvoir en opposition avec les pouvoirs institués.

\footnotetext{
${ }^{13} \mathrm{~J}$. Wresinski, cité dans medicitoyen.org, ATD Quart Monde et la démocratie participative.

${ }^{14}$ P. Brun, «Croisement des savoirs et pouvoir des acteurs », V.S.T, vol. 4, n 76,2002 , p. 55-60.

${ }^{15}$ H. Balazard, Agir en démocratie, L’Atelier, Paris, 2015.

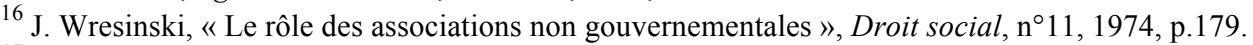

${ }^{17}$ Salarié de l'institut de recherche, entretien, 21 avril 2015.
} 


\section{ATD Quart Monde : du community organizing post-alinskien ?}

Alors que l'idée initiale de Saul Alinsky est de s'inspirer du syndicalisme en créant des contrepouvoirs dans la société civile, Joseph Wresinski s'intéresse, quant à lui, au «sous-prolétariat» délaissé par le marxisme et le mouvement ouvrier. Il s'agit ainsi de revisiter le modèle des syndicats en reprenant leur rôle là où ces derniers n'agissent pas. C'est au service de cet effort d'organisation des plus pauvres que se situe dans la vision de Wresinski le compagnonnage avec des figures comme Geneviève Anthonioz de Gaulle ${ }^{18}$. Dans une optique pragmatique, cette alliance a pour but de construire un contre-pouvoir incontournable sur la scène publique. ATD Quart Monde se déploie alors en un réseau de groupes locaux dont le but est de mobiliser des «militants quart monde » et des « alliés ». Ce réseau se rassemble ensuite autour d'un porte-parole national pour mener des campagnes politiques spécifiques.

Plutôt que les réseaux sociaux organisationnels (églises, associations, écoles, etc.) utilisés par Alinsky pour mobiliser les citoyens, les membres d'ATD Quart Monde préfèrent les réseaux individuels afin de pouvoir atteindre les personnes les plus isolées socialement qui participent rarement aux actions collectives. Comme le souligne Hélène Balazard, une des limites du broad-based community organizing est qu'en s'appuyant sur les communautés déjà constituées, il n'arrive pas à atteindre ces personnes ${ }^{19}$. Pour sa part, le mouvement ATD Quart Monde focalise son action sur la mobilisation des acteurs faibles à travers le dispositif de l'université populaire ${ }^{20}$. Concrètement, il s'agit de créer un lien de confiance sur la durée. Prenons deux exemples pour illustrer cette stratégie. Premièrement, un animateur se sert de son bénévolat à la soupe populaire pour atteindre certaines personnes isolées socialement et leur proposer de venir à l'université populaire. Deuxièmement, un animateur organise ses réunions dans un foyer de jour pour sans domicile fixe, il vient également une fois par semaine pour développer des liens et expliquer la démarche de l'université populaire. Le mode d'action d'ATD Quart Monde se rapproche en cela de ce que Julien Talpin appelle le community organizing postalinskien qui utilise les réseaux individuels afin d'organiser les «non-organisés » et en particulier les plus défavorisés ${ }^{21}$.

Ce qui semble opposer le plus Wresinski à Alinsky réside dans le rapport entretenu avec les pouvoirs publics. Saul Alinsky défend un modèle de pression fondé sur le conflit. L'autonomie par rapport à toute forme de pouvoir est alors érigée en principe absolu. Joseph Wresinski a lui aussi fait du community organizing dans le camp de Noisy-Le-Grand en organisant les habitants en opposition aux pouvoirs publics. Mais, par la suite, il adopte une vision plus nuancée par rapport au conflit, en

\footnotetext{
${ }^{18}$ Nièce de Charles de Gaulle, figure de la résistance française.

${ }^{19}$ H. Balazard, op. cit.

${ }^{20}$ A. Roy, La mobilisation des acteurs faibles, Mémoire de master en urbanisme, IUL, Lyon, 2014.

${ }^{21}$ J. Talpin, « Mobiliser les quartiers populaires », La vie des idées, 2013.
} 
valorisant davantage la collaboration. Les relations entre le mouvement et les pouvoirs publics peuvent alors être analysées comme une « coopération conflictuelle », pour reprendre l'expression de Pierre Dommergues ${ }^{22}$. Cette posture suppose une stratégie fondée sur une collaboration critique dans laquelle l'alliance est privilégiée bien que le recours au conflit puisse être envisagé pour augmenter son pouvoir d'influence. Pour Joseph Wresinski et son mouvement, la constitution d'un réseau d'alliance est considérée comme essentielle à la fois pour leur stratégie d'émancipation collective, mais également pour augmenter la puissance d'influence d'un mouvement qui organise des personnes à faibles ressources. Cependant, cette stratégie n'empêche pas d'entrer en conflit avec les pouvoirs publics. Si la coopération est souvent mise en avant, ATD Quart Monde entre régulièrement à l'échelle nationale dans le registre de la dénonciation, voire de la pression : "On doit garder cet équilibre entre la dénonciation des humiliations dont les plus pauvres sont les victimes et chercher à coopérer avec les pouvoirs publics. Le plus important c'est la liberté c'est-à-dire qu'aucune coopération avec les pouvoirs publics ne nous fera taire, aucune ${ }^{23}$ !» Notons pourtant que, dans une optique pragmatique, Saul Alinsky valorise également le compromis, la négociation et l'alliance. Si, les pouvoirs publics sont considérés comme faisant partie des «oppresseurs », il s'agit tout de même d'agir à l'intérieur du système en tirant parti de toutes les opportunités. Finalement, la posture pragmatique radicale d'Alinsky ne serait-elle pas comparable avec l'approche de collaboration critique et réflexive de Wresinski?

Le mode d'action d'ATD Quart Monde, forme de community organizing post-alinskien, peut être illustré à partir de l'exemple de la campagne contre les discriminations pour origines sociales menée actuellement. Cette campagne fait écho à la vision de Joseph Wresinski qui affirmait en 1973 que les personnes en situation d'extrême pauvreté « sont parmi ces minorités victimes de discrimination de droit $^{24} \gg$. Le délégué national d'ATD Quart Monde reprend l'idée en 2009 et lance un dispositif de vérification de l'hypothèse et de capitalisation de la connaissance auprès de la base militante via la sollicitation des groupes locaux et des universités populaires. À partir de cette enquête qualitative et d'une alliance avec un centre de recherche pour effectuer une étude quantitative, ATD Quart Monde entend alors étayer scientifiquement cette thèse. Avec la publication d'un rapport de recherche, le mouvement entre dans la phase de communication et d'interpellation des pouvoirs publics. Le mode d'action est varié : pétition, manifestation publique, dénonciation médiatique de cas de discrimination, construction d'un réseau d'alliance associatif, interpellation des défenseurs des droits, alliance avec certaines personnalités politiques, etc. Aujourd'hui, une proposition de loi pour faire reconnaître la précarité économique comme un critère de discrimination a été adoptée par le Sénat et attend d'être

\footnotetext{
${ }^{22}$ P. Dommergues (dir.), La société de partenariat, AFNOR : Anthropos, Paris, 1988.

${ }^{23}$ B. Tardieu, op. cit.

${ }^{24}$ J. Wresinski, op. cit., 2007, p. 154.
} 
examinée à l'Assemblée nationale ${ }^{25}$. À l'image du rôle d'ATD Quart Monde dans la prise en compte du problème de l'illettrisme dans les années $1970-1980^{26}$ ou dans l'adoption de la loi d'orientation relative à la lutte contre les exclusions de 1988, l'exemple de cette campagne contre les discriminations pour cause de pauvreté illustre en quoi la posture de « coopération conflictuelle » peut être à l'origine d'innovations politiques.

\section{Conclusion}

Pour conclure, alors que beaucoup s'interrogent sur la possibilité de transposer l'empowerment radical en France, une figure française comme celle de Joseph Wresinski est trop peu prise en compte. Pourtant, à l'instar de Paulo Freire et de Saul Alinsky, Joseph Wresinski a construit, dès les années 1960, une stratégie originale d'émancipation radicale qui mériterait plus d'attention dans la perspective d'une sociologie de l'empowerment. Par ailleurs, alors que des auteurs comme Marie Hélène Bacqué et Carole Biewener évoquent les résistances culturelles et politiques au paradigme de l'empowerment ${ }^{27}$, une analyse sociohistorique de ce mouvement pionnier permettrait d'éclairer les conditions françaises d'émergence et de pérennisation d'un projet d'émancipation radical et de sa portée subversive.

\section{L'auteur}

\section{Alex Roy}

Chercheur en sociologie urbaine, associé au laboratoire Environnement, ville et société (EVS), composante Recherches interdisciplinaires Ville, Espace, Société (RIVES), UMR 5600 .

Thèmes de recherche: empowerment des personnes en situation de pauvreté ; démocratie délibérative et participative ; mouvements sociaux ; transition socio-écologique.

THESE : ROY A., 2019, De l'infrapolitique à la révolution démocratique : ethnographie culturelle du mouvement ATD Quart Monde, Thèse de doctorat en géographie, aménagement et urbanisme, Vaulxen-Velin, Université Lumière Lyon 2, ENTPE.

\footnotetext{
${ }^{25}$ Texte adopté par le sénat $n^{\circ} 114$, le 18 juin 2015 .

${ }^{26}$ B. Lahire, op. cit.

${ }^{27}$ M.-H. Bacqué et C. Biewener, op. cit.
} 